\title{
Dual Branch-Promoting and Branch-Repelling Actions of Slit/Robo Signaling on Peripheral and Central Branches of Developing Sensory Axons
}

\author{
Le $\mathrm{Ma}^{1,2}$ and Marc Tessier-Lavigne $\mathrm{e}^{1,3}$ \\ ${ }^{1}$ Howard Hughes Medical Institute, Department of Biological Sciences, Stanford University, Stanford, California 94305, 2Department of Cell and \\ Neurobiology, Zilkha Neurogenetic Institute, Keck School of Medicine, University of Southern California, Los Angeles, California 90089, and ${ }^{3}$ Division of \\ Research, Genentech, South San Francisco, California 94080
}

\begin{abstract}
Secreted Slit proteins signal through Robo receptors and negatively regulate axon guidance and cell migration, but in vertebrates, Slit proteins can also stimulate branching and elongation of sensory axons and cortical dendrites in vitro. Here, we show that this branching activity is required for developing peripheral sensory arbors in vivo, because trigeminal sensory branching above the eye is reduced in Slit2;Slit3 double or Slit1,2,3 triple mutants. A similar phenotype is observed in Robo1;Robo2 double mutants, implicating Robo receptors in mediating this activity. Interestingly, by studying the central projection of sensory neurons in the spinal cord, we discovered that Slit ligands are also required for proper guidance of sensory branches during bifurcation but through a different cellular mechanism. In Slit1;Slit2 or Robo1;Robo2 double mutant mice, sensory axons enter the spinal cord prematurely because of the loss of an inhibitory guidance function on one of the daughter branches of each afferent during bifurcation. Together, our studies reveal that Slit/Robo signaling contributes to patterning both the peripheral and central branches of sensory neurons but via distinct positive branching and negative guidance actions, respectively.
\end{abstract}

Key words: axon branching; Slit; Robo; arborization; bifurcation; sensory neuron

\section{Introduction}

Neuronal dendrites and axons are usually branched. Some form just two daughter branches from a common neurite, some sprout multiple collaterals from a main shaft, and yet others exhibit elaborated arbors, often at nerve terminals, that can number in the hundreds or even thousands. These branched structures are formed throughout development, and their formation is tailored to the functional needs of the neural networks they assemble.

Despite their importance, our understanding of the molecular mechanisms controlling branching is fragmentary, although biochemical studies and genetic screens have begun to identify the molecules involved. Inside the cell, genetic programs controlled by transcription factors have been found to regulate branch formation (Livet et al., 2002; Parrish et al., 2006). Outside the cell, environmental factors have been proposed to regulate many branching events in the brain. Some molecules with positive influence on neurite growth and guidance can stimulate branch

Received April 2, 2007; revised May 3, 2007; accepted May 7, 2007.

This work was supported by Damon Runyon Cancer Research Foundation Grant DRG-\#1607 (L.M.) and by the Howard Hughes Medical Institute (M.T.-L.). We thank A. Plump, H. Long, C. Sabatier, A. Yaron, F. Wang, and other members of the Tessier-Lavigne laboratory for helpful discussions and for sharing expertise and reagents, and we thank H. Lin for technical assistance. We thank Louis Reichardt for the TrkA antibody, David Ornitz for the Slit3 mouse, and Whit Tao and Mu-ming Poo for help with Dil iontophoresis. We also thank Tom Jessell for critical comments on this manuscript.

Correspondence should be addressed to Marc Tessier-Lavigne, Division of Research, Genentech, 1 DNA Way, South San Francisco, CA 94080. E-mail: marct|@gene.com.

D0I:10.1523/JNEUROSCI.1479-07.2007

Copyright $\odot 2007$ Society for Neuroscience $\quad$ 0270-6474/07/276843-09\$15.00/0 formation. For example, neurotrophins, best known for their dual role in supporting neuronal survival and promoting neurite outgrowth, have been implicated in branching (Hoyle et al., 1993; Cohen-Cory and Fraser, 1995; Lentz et al., 1999). Other positive candidates are the secreted protein Anosmin, B class Ephrins, and Wnts (Yates et al., 2001; Krylova et al., 2002; Soussi-Yanicostas et al., 2002). Conversely, factors with inhibitory effects on neurite growth and guidance can negatively regulate branch formation and stability. For example, Semaphorin3F has been implicated in pruning of preexisting branches during hippocampal development (Bagri et al., 2003).

Our previous study implicated the extracellular protein Slit2 in regulating sensory axon branching (Wang et al., 1999). Using dorsal root ganglion (DRG) cell cultures, we found that an $\mathrm{N}$-terminal fragment of Slit2 (Slit2N) stimulated branching and elongation of rat DRG axons from embryonic day 14 (E14) when they normally arborize in their targets in vivo. Evidence to support this branching effect was also obtained using a trigeminal ganglion explant culture (Ozdinler and Erzurumlu, 2002) and by misexpression in zebrafish (Yeo et al., 2004). In the CNS, Slit1 was shown to regulate cortical dendritic branching in vitro (Whitford et al., 2002). Furthermore, Slits have been implicated in axonal repulsion, a function that is conserved in flies, worms, and vertebrates, and mediated by the Robo family of receptors; this function has been validated in numerous genetic studies (Kidd et al., 1998, 1999; Zallen et al., 1998; Fricke et al., 2001; Hao et al., 2001; Plump et al., 2002; Long et al., 2004). In contrast, no loss- 
of-function genetic data have been provided to validate the branching activity of Slits. In this study, we characterize the in vivo role of Slits and Robos and provide genetic evidence to support the involvement of Slits in regulating peripheral arborization of sensory neurons via Robos. Surprisingly, we also found that the negative guidance activity of Slits is also used to regulate proper bifurcation of the central sensory afferents at an earlier stage. These results indicate that a single signaling pathway can be used in different branching processes via distinct positive and negative actions.

\section{Materials and Methods}

Mouse strains. Mice were handled according to the protocols approved by the Institutional Animal Care and Use Committees at Stanford University and the University of Southern California following the National Institutes of Health regulations. All animals were generated in a mixed CD-1/129Sv/C57BL/6 background. To generate Slit mutants, we crossed Slit $^{-/-} ;$Slit $^{+/-}$, Slit $_{2}^{+/-} ;$Slit $^{+/-}$or Slit $1^{-/-} ;$Slit $^{+/-} ;$Slit $^{+/-}$animals and obtained the desired double or triple mutants with a frequency of one in four or 1 in 16. Robo mutants were obtained by crossing double heterozygous Robo1;Robo2 animals, which have the two mutant alleles (1.8 megabases apart) already linked to the same chromosome 16. Genotyping of Slit1, Slit2, Slit3, Robo1, and Robo2 was done by PCR as described previously (Plump et al., 2002; Yuan et al., 2003; Grieshammer et al., 2004; Sabatier et al., 2004).

Immunohistochemistry and microscopy. For immunostaining in tissue sections, embryos were fixed with $4 \%$ paraformaldehyde (PFA) in PBS equilibrated with $30 \%$ sucrose in PBS and frozen in the OCT embedding medium (Tissue-Tek; Miles, Elkhart, IN). Following a common protocol (Kennedy et al., 1994; Serafini et al., 1996), thin sections (16 $\mu \mathrm{m})$ were cut on a cryostat (Leica, Nussloch, Germany) and stained in a buffer containing PBS, $0.1 \%$ Triton X-100, 10\% goat serum. Monoclonal antibodies against TAG-1 (transiently expressed glycoprotein; antibody 4D7; $1: 200)$ and neurofilament (2H3; 1:200) were obtained from the Developmental Studies Hybridoma Bank (University of Iowa, Iowa City, IA), and the rabbit polyclonal antibody against tyrosine receptor kinase A (TrkA) (1:500) was kindly provided by Louis Reichardt (University of California, San Francisco, San Francisco, CA). Fluorescent secondary antibodies (1:200 dilution) conjugated with Cy2 or Cy3 (Jackson ImmunoResearch, West Grove, PA) were used for imaging on an Axioplan2 compound microscope (Zeiss, Thornwood, NY) equipped with a digital camera [Spot II-RT (Diagnostic Instruments, Sterling Heights, MI) or AxioCam (Zeiss)].

Whole-mount staining for neurofilament on embryos was performed using a monoclonal antibody $(2 \mathrm{H} 3 ; 1: 200)$, peroxidase-conjugated secondary antibodies (Chemicon, Temecula, CA), and diaminobenzadine (Sigma, St. Louis, MO) as the substrate (Cheng et al., 2001). After staining, younger embryos (E10.5) were cleared with a mixture of benzyl alcohol and benzyl benzonate (1:2). Photographs were taken on a stereoscope (Stemi 11; Zeiss) equipped with a Spot II-RT digital color camera.

In situ hybridization. Cryosections $(16 \mu \mathrm{m})$ of E12.5 rat embryos were processed for in situ analysis following a published procedure (Serafini et al., 1996). ${ }^{35}$ S-labeled probes were generated using the following published templates: Slit1, Slit2, Slit3, Robo1, Robo2, and Rig1 (Brose et al., 1999). Dark-field images were obtained from an Axioplan2 microscope (Zeiss)

DiI labeling. To label axonal tracts in the PFA-fixed spinal cord, a small puff of $0.2 \%$ DiI (Invitrogen, San Diego, CA) dissolved in ethanol was injected into attached DRGs using a glass pipette with a 5-10 $\mu \mathrm{m}$ opening. The tissue was incubated at $37^{\circ} \mathrm{C}$ for $2 \mathrm{~d}$ and then sectioned with a vibratome (Leica) at $50 \mu \mathrm{m}$ thickness to allow higher resolution visualization of sensory collaterals in the spinal cord. For single-cell labeling, a small dye crystal was delivered to the DRG from an ethanol solution of DiI $(0.5 \%)$ in a glass pipette ( $1 \mu \mathrm{m}$ opening) by iontophoresis with a current of $\sim 5-20 \mathrm{nA}$. The dye was allowed to diffuse at $25^{\circ} \mathrm{C}$ overnight before the labeled axons were directly visualized and photographed from the lateral side of the spinal cord. All images were taken on an Axioplan2 microscope (Zeiss).
In vitro growth cone collapse assay. DRG explants isolated from E12.5 rat embryos were plated in eight-well chamber slides (Labtek, Andover, MA) coated with poly-D-lysine $(10 \mu \mathrm{g} / \mathrm{ml})$ and laminin $(10 \mu \mathrm{g} / \mathrm{ml}) \mathrm{se}-$ quentially and cultured in an F-12 medium (Invitrogen) with the N3 supplement, $40 \mathrm{~mm}$ glucose, $0.5 \%$ fetal calf serum plus the nerve growth factor (NGF; $25 \mu \mathrm{g} / \mathrm{ml}$; Sigma), or NT-3 (25 $\mu \mathrm{g} / \mathrm{ml}$; PeproTech, Rocky Hill, NJ). After $16 \mathrm{~h}$, they were treated with control buffer, S2N, or Sema3A for different time periods and fixed for rhodamine-phalloidin staining. Recombinant Slit2N was purified from COS cells and used at 1 $\mu \mathrm{g} / \mathrm{ml}$, approximately threefold higher than the concentration that could elicit E14 neuron branching in collagen gels (Wang et al., 1999). Sema3A was from the concentrated medium of COS cells transiently expressing the protein. For time-lapse movies, cells cultured on glass coverslips were transferred to a custom-made chamber and cultured at $37^{\circ} \mathrm{C}$ on an Axivert 100 microscope. They were subsequently treated with Slit $2 \mathrm{~N}$ proteins $(1 \mu \mathrm{g} / \mathrm{ml})$, and their behaviors were recorded using a Dage-MTI (Michigan City, IN) video camera at 5 s intervals.

\section{Results}

Slit/Robo signaling is required for peripheral arbor formation of sensory neurons in mice

To determine whether Slits and Robos play a role in controlling branching morphogenesis of sensory axons in vivo, we studied mouse mutants for all three Slit genes and two of the Robo genes [the third Robo gene, Robo3/Rig1, is not expressed by sensory neurons (supplemental Figs. 1, 2, available at www.jneurosci.org as supplemental material) and will not be considered further].

We first examined the roles of Slits in the formation of peripheral arbors, because they most resemble the in vitro branched morphology stimulated by Slit2N (Wang et al., 1999). We examined embryos with mutations in the Slit1, Slit2, and Slit3 genes using neurofilament immunostaining in whole-mount embryos to follow the peripheral projections at different ages and locations. There was a subtle change in the branching pattern of the peripheral projections of DRG sensory neurons in the trunk and limb (data not shown), but we found a dramatic defect in the ophthalmic projection from the trigeminal sensory ganglion in the head. This projection normally has two characteristic and highly branched arbors, one above the eye and one below, in wild-type or heterozygous animals $(n=9)$ (Fig. $1 A)$. These two arbors start to grow out at approximately E11.5 and become fully developed, covering the entire face at E13.5 (data not shown). At E12.5, when they begin to mature, the top structure has five to six radially extended branches, whereas the bottom one grows two main stems that project in opposite directions. Although the overall size of these two structures varies even among the wildtype or Slit2;Slit3 heterozygous controls from the same litter, the top arbor is always significantly larger than the bottom one (Fig. $1 A)$. This can be demonstrated clearly by the ratio of the area occupied by the top versus the bottom, which is $1.41 \pm 0.24$ for the wild type (Fig. 1C).

As shown by RNA in situ hybridization in rat (supplemental Figs. $1 A-C, 3 A$, available at www.jneurosci.org as supplemental material) as well as in mouse (data not shown) embryos, Slit2 and Slit3 are highly expressed around the eye in E13 rat and E11.5 mouse embryos (which represent developmentally equivalent stages in the two species). Because Slit1 is not detected there, we first examined Slit2;Slit3 mutant embryos $(n=4)$. We found that the bottom structure was unaffected in the mutant, but the top structure was dramatically reduced in size when compared with stage-matched littermates (Fig. $1 B$ ). The ratio of the top and the bottom branching areas was reduced by twofold to $0.69 \pm 0.30$ in the double mutants (Fig. 1C), and this reduction reflects a change in both branching and neurite growth, because the ratio of the 

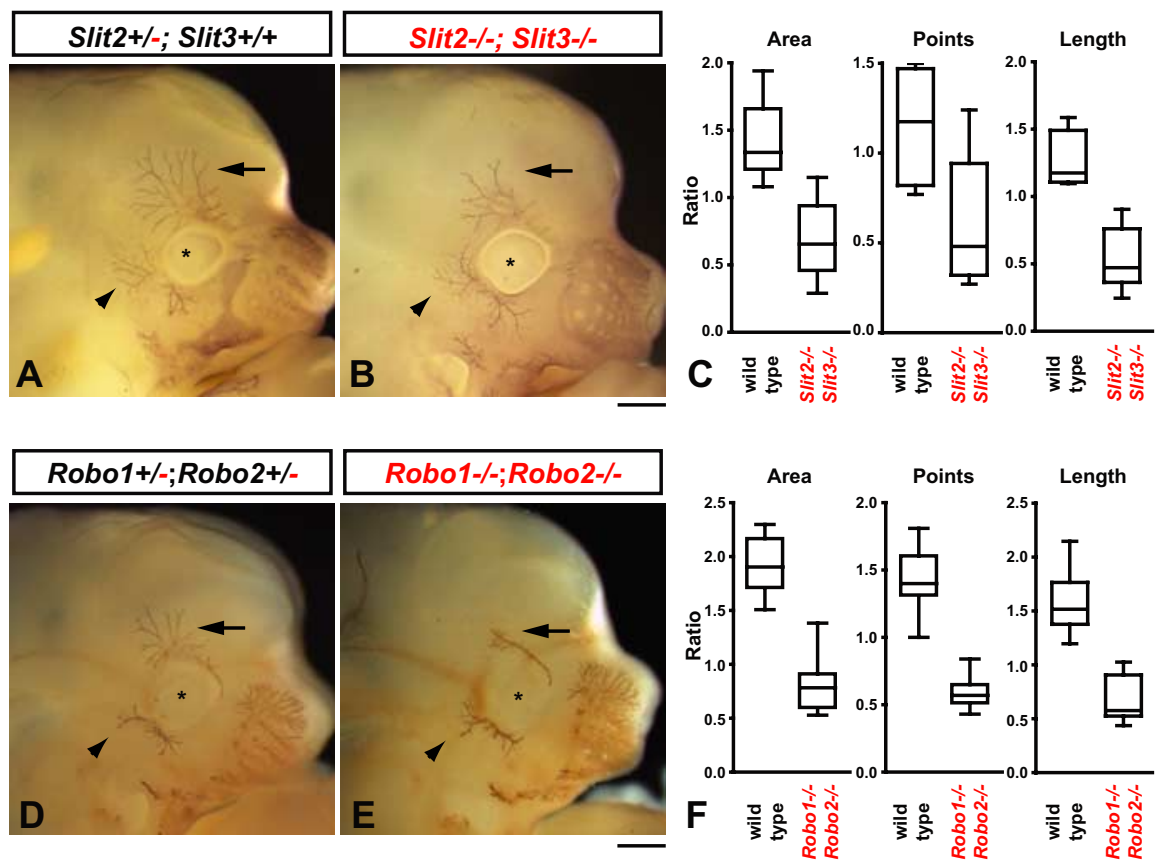

Figure 1. Reduction in peripheral aborization of the ophthalmic projection from trigeminal ganglia in the Slit2;Slit3 or the Rob01;Robo2 double mutant. $\boldsymbol{A}, \boldsymbol{B}$, The ophthalmic projections of the trigeminal ganglion from E12.5 embryos were visualized by neurofilament immunostaining, which revealed the two ophthalmic branches surrounding the eye $\left.{ }^{*}\right)$. When compared with its littermate control from the Slit2; Slit3 cross ( $\boldsymbol{B}$ vs $\boldsymbol{A}$ ), the top branched structure (arrows) is greatly reduced in the double mutant, whereas the bottom one (arrowheads) remains the same. Because of the variation in the overall growth of the two branched structures even in the same litter, a Slit2 ${ }^{+/-} ; \mathrm{Slit}^{+/+}$is shown here as a littermate control to match the bottom arbor in the double mutant. Scale bars, $50 \mu \mathrm{m}$. C, The extent of branching in the wild-type or the Slitz; Slit3 mutant animals is quantified by measuring the area occupied by the branched arbors, the number of branching points, or the total branch length. To normalize for slight differences in developmental stages, both are plotted as the ratio of the top arbor versus the bottom one, which appears to follow a normal developmental course in all animals. The ratio of the area, points, or lengths in the wild type is $1.41 \pm 0.24$, $1.16 \pm 0.34$, or $1.27 \pm 0.22$, respectively, whereas the ratio in the mutant is $0.69 \pm 0.30,0.61 \pm 0.36$, or $0.56 \pm 0.23$, respectively. $\boldsymbol{D}, \boldsymbol{E}$, The ophthalmic projections from the Robo1;Robo2 cross are stained with neurofilament antibodies as in $\boldsymbol{A}$ and $\boldsymbol{B}$. The embryos shown here are slightly younger than those in $\boldsymbol{A}$ and $\boldsymbol{B}$ to illustrate the requirement of Robo signaling in early branch formation. Scale bars, $500 \mu \mathrm{m}$. $\boldsymbol{F}$, Quantification of the difference in Robo1; Robo2 animals as in $\boldsymbol{C}$. The ratio of branching area, points, or length drops from $1.94 \pm 0.26,1.42 \pm 0.22$, or $1.57 \pm 0.28$ in the wild type to $0.79 \pm 0.22,0.59 \pm 0.11$, or $0.71 \pm 0.21$ in the mutant, respectively.
We next examined whether members of the Robo family are also involved in peripheral arborization in vivo. Both Robol and Robo2 have been shown previously to be dynamically expressed in the DRG and trigeminal ganglion (Brose et al., 1999). We confirmed by RNA in situ hybridization that they are expressed in both ganglia at the time when sensory axons are making branches in rat embryos (supplemental Figs. $1 D-F, 2 D-F$, available at www.jneurosci.org as supplemental material) as well as in mouse embryos (data not shown). Therefore, we generated Robo1 ${ }^{-1}$ -;Robo2 ${ }^{-1-}$ double mutants and examined the peripheral branching pattern from both DRGs and trigeminal ganglia, using neurofilament immunostaining. As in the compound Slit mutants, ophthalmic arbors from the trigeminal ganglion were greatly reduced in the $R_{0 b o 1^{-1-}}$; Robo2 ${ }^{-/-}$mutant $(n=12)$, because the ratio of branching area, branching point numbers, or total branch lengths dropped by 2.2 -fold to 2.4 -fold, from $1.94 \pm 0.26$, $1.42 \pm 0.22$, or $1.57 \pm 0.28$ in the wild type to $0.79 \pm 0.22,0.59 \pm 0.11$, or $0.71 \pm 0.21$ in the mutant (Fig. $1 F$ ). The sample embryos shown here (Fig. $1 D, E$ ) are younger than those in Figure 1, $A$ and $B$, and demonstrate an even more dramatic reduction in the mutant. This indicates again a key requirement of Robo signaling for early arbor formation in this branch.

Together, these results support a role for Slit proteins, particularly Slit2 and Slit3, in stimulating the branching of sensory axons in vivo and also suggest that this branching activity is mediated by Robol and Robo2. number of branching points or the ratio of branch lengths is also reduced to $0.61 \pm 0.36$ or $0.56 \pm 0.23$, respectively, in the mutant from $1.16 \pm 0.34$ or $1.27 \pm 0.22$ in the wild type (Fig. $1 C$ ).

To avoid the possibility that Slit1 may have compensated for the loss of the other two Slits, we also examined the triple Slit1; Slit2;Slit3 mutant $(n=2)$. The embryos were developmentally delayed by a few hours compared with those shown above, even when they were collected at the same time of day, and the overall size of the ophthalmic branches was, as a consequence, proportionately smaller. Interestingly, the top branched structure was completely lost in the triple mutant, whereas the bottom one remained unchanged when compared with the littermate control (supplemental Fig. 3B, available at www.jneurosci.org as supplemental material). We compared the branching ratio in different Slit2;Slit3 genotypes in the wild-type (data not shown) or Slit1 mutant background and found that both the arbor size and the branch point number are sensitive to the loss of wild-type Slit alleles, with a minimum of two copies of either Slit2 or Slit3 needed to maintain the normal pattern (supplemental Fig. $3 C, D$, available at www.jneurosci.org as supplemental material). These results are consistent with a role for Slit proteins, particularly Slit2 and Slit3, in stimulating one of the trigeminal sensory branches.

\section{Abnormal guidance of central branches of DRG neurons in Slit and Robo mutants}

We next examined whether Slit/Robo signaling affects the projection or branching of central branches of sensory axons. As described, both Robol and Robo2 are expressed in sensory neurons; in addition, two of the three Slit genes, Slit1 and Slit2, are expressed in the dorsal spinal cord, right alongside the dorsal root entry zone (DREZ), at the developmental stage (E12.5) when collaterals start to sprout (Brose et al., 1999) (supplemental Fig. $2 A-C$, available at www.jneurosci.org as supplemental material). Therefore, we hypothesized that collaterals might also be regulated by Slits via their Robo receptors.

To test this hypothesis in the Slit and Robo mutant mice, we first analyzed E13.5 embryos by implanting a DiI crystal in the DRG to label their central projections (Fig. 2A). This tracing method allowed us to assess qualitatively whether the collaterals were still formed in the absence of Slit/Robo signaling (Ozaki and Snider, 1997). As shown in the cross-sections of labeled spinal cords, collaterals sprout from the dorsal funiculus and enter the gray matter in the wild-type or the Slit $1^{+/-} ;$Slit $2^{+/-}$animals used as controls (Fig. $2 \mathrm{~B}, \mathrm{C}$, arrowheads). At this age, the collaterals are mainly from muscle afferents, which follow a hyperbolic trajec- 

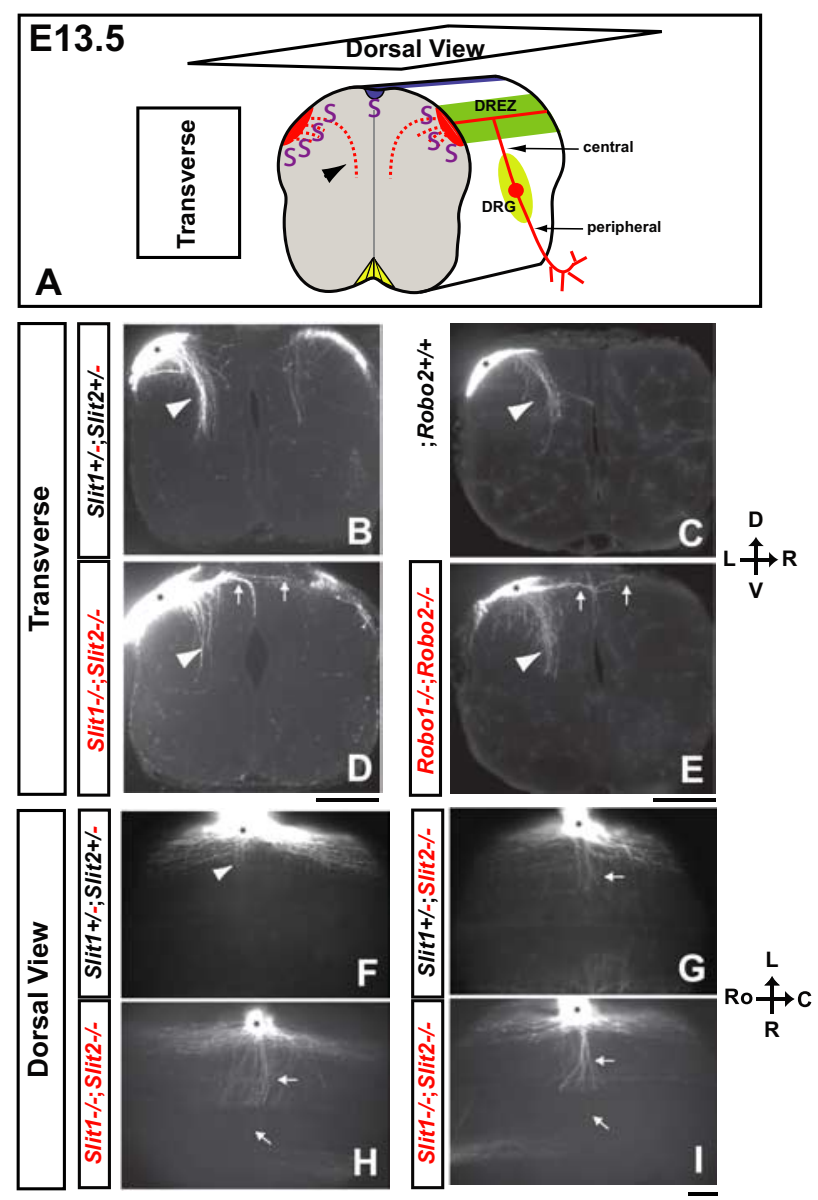

Figure 2. Misprojections of sensory afferents into the spinal cord are revealed by Dil labeling in Slit1 $1^{-1-}$;Slit2 $2^{-/-}$or Robo1 ${ }^{-/-} ;$Robo2 $2^{-/-}$mutant embryo. A, A schematic diagram showing the central and peripheral projections of sensory axons in the spinal cord at E13.5. The viewing planes of Dil labeling shown in $\boldsymbol{B}-\boldsymbol{E}$ and $\boldsymbol{F}-\boldsymbol{I}$ are indicated by the boxes around the diagram. Slit expression is indicated by the letter S. $\boldsymbol{B}-\boldsymbol{E}$, Central branches labeled with Dil injected into the DRG were visualized in transverse sections of E13.5 spinal cords from the site of dorsal root entry. In either the wild-type $(\boldsymbol{C})$ or the Slit ${ }^{+/-} ;$Slit2 $2^{+/-}(\boldsymbol{B})$ animals, collaterals of the la muscle afferents (arrowheads) emerge from the dorsal funiculus $\left({ }^{*}\right)$, the bundled axons that extend along the longitudinal tract. These collaterals are also present in their littermate mutants $(\boldsymbol{D}, \boldsymbol{E})$, although their hyperbolic trajectory is slightly altered. In addition, in the most dorsal side of the spinal cord, bundles of labeled axons were found extending inside the spinal cord toward the midline. Some of these overshooting fibers (arrows) turn ventrally at the midline, whereas others venture across the midline and extend on the contralateral side of the spinal cord. L, Left; R, right; D, dorsal; V, ventral. Scale bars, $100 \mu \mathrm{m}$. F-I, DRG projections labeled by Dil were visualized from the dorsal side of the spinal cord. In the E13.5 Slit ${ }^{+/-}$; Slit2 ${ }^{+/-}$embryo $(\boldsymbol{F})$, the labeled sensory afferents are present in the DREZ along the rostrocaudal axis. A small number of collaterals (arrowheads) just begin to enter the spinal cord and remain close to the DREZ. However, in either the Slit $1^{+/-} ;$Slit2 $^{-/-}(\boldsymbol{G})$ or Slit1 ${ }^{-/-} ;$Slit2 ${ }^{-/-}$ $(\boldsymbol{H}, \boldsymbol{I})$ spinal cord from the same age, bundles of axons (arrows) leave the longitudinal tract and extend toward the midline of the spinal cord. Most of the misprojections appear to extend directly from the dorsal roots $\left({ }^{*}\right)$, whereas some grow out from more distal regions ( $\boldsymbol{H}$, arrows). In addition, as shown in $\boldsymbol{D}$, many overshooting fibers stop at the midline, but occasionally one or two (oblique arrows in $\boldsymbol{H}$ and $\boldsymbol{I}$ ) reach the other side of the spinal cord. L, Left; R, right; Ro, rostral; C, caudal. Scale bar, $100 \mu \mathrm{m}$.

tory to project ventrally, as described previously (Ozaki and Snider, 1997). When we analyzed the mutant littermates of the control animals, we observed that the collaterals still formed and projected in the gray matter of the Slit $1^{-/-} ;$Slit $^{-/-}$double mutant $(n=4)$ or the Robo1 ${ }^{-/-} ;$Robo $^{-/-}$double mutant $(n=3)$ (Fig. $3 D, E$, arrowheads). Their hyperbolic trajectory was still evident, although it was slightly altered (likely because of a problem described below). We also examined the collaterals from the small-diameter cutaneous fibers that are more prominent in E14.5 animals and found no qualitative change in their formation, location, or lamina termination (supplemental Fig. 4, small arrowheads). This shows that the formation of DRG central collaterals is not dependent on Slit/Robo signaling.

Although DRG central collaterals still form in the absence of Slit/Robo signaling, we found an unexpected misprojection of DRG axons inside the spinal cord of these mutants. This was first evident when the DiI-labeled whole-mount E13.5 spinal cord was viewed from the dorsal side. In the wild-type (data not shown) or the Slit1 $1^{+/-}$Slit2 ${ }^{+/-}$(Fig. 2F) control animals, DiI-labeled DRG axons bifurcated normally at the DREZ and extended in the opposite direction along the rostrocaudal axis. At this age, only a few short nascent collaterals appear to develop from the longitudinal axons and project into the spinal cord; they can be recognized as out-of-focus fibers in the image (Fig. $2 F$, arrows). In the Slit $1^{-/-}$; Slit $2^{-1-}$ double mutant, however, more and longer DiI-labeled fibers can be seen leaving the DREZ, often at the site of bifurcation, and projecting toward the midline (Fig. $2 \mathrm{H}, \mathrm{I}$, arrows). As seen most clearly in transverse sections of both the Slit $1^{-1-} ;$ Slit $2^{-/-}$and the Robo1 ${ }^{-/-} ;$Robo $^{-/-}$mutants, these overshooting axons can often be found extending in the dorsal horn, a few cell layers below the dorsal surface. Most of them stop at the ventricular zone, but a small number cross the midline, right below the roof plate and above the ventricle (Fig. $2 D, E$, arrows).

Thus, loss of Slit/Robo signaling does not appear to affect the sensory collaterals but rather leads to an abnormal projection of fibers inside the spinal cord.

\section{The central afferent defects occur early during bifurcation}

The defect in the central projections of sensory neurons described above could result from premature formation of collaterals. Alternatively, the overshooting fibers inside the spinal cord could directly come from misregulated growth of primary axons or branches that normally stay outside the gray matter of the spinal cord. To distinguish these two possibilities, we studied the phenotype by examining axonal markers that can distinguish different segments of sensory axons and different neuronal populations, as well as determining the earliest time when the defect occurs.

The axonal surface marker TAG-1 has been shown to label mainly primary sensory afferents and bifurcated branches in the DREZ, and it is downregulated in rat embryos after E14 (equivalent to E12.5 of the mouse) when their collaterals start to enter the spinal cord (Dodd et al., 1988; Furley et al., 1990) (Fig. 3A). Interestingly, in E11.5 mouse embryos from the Robo1 ${ }^{-1-}$; Robo2 $2^{-1-}$ or the Slit $1^{-1-}$;Slit $2^{-/-}$animal $(n>3)$, the overshooting axons in the dorsal spinal cord already expressed TAG-1 and appeared to continue from the dorsal root (Fig. $3 B, C$, arrows), suggesting that they are likely either primary axons or bifurcated branches that inappropriately entered the spinal cord, rather than collaterals. These fibers were also labeled by antibodies against neurofilament (Fig. 3E,F, arrows), and a small number were TrkA positive (supplemental Fig. 6, available at www.jneurosci. org as supplemental material), suggesting that the defect is not restricted to any specific subpopulation of DRG neurons. A similar defect was also observed for the central projection of trigeminal neurons (supplemental Fig. 5, available at www.jneurosci.org as supplemental material).

These overshooting axons can be seen at very early stages, starting from E10.5, when sensory axons just reach the spinal cord and begin to bifurcate to form the dorsal funiculus. This can 

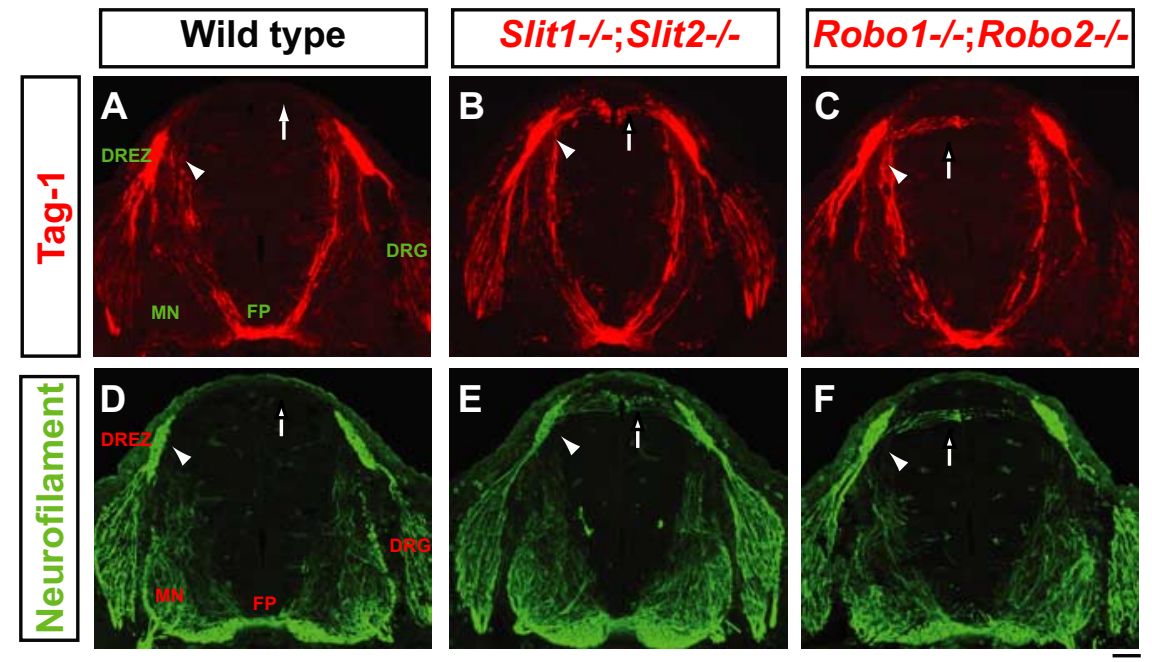

Figure 3. Axon misprojections visualized by TAG-1 and neurofilament immunostaining. Transverse sections of the spinal cord from the brachial region of E11.5 embryos of different genotypes were stained with antibodies against TAG-1 ( $\boldsymbol{A}-\boldsymbol{C})$ or neurofila-

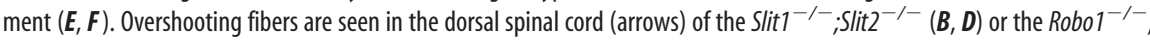
Robo2 $^{-/-}(\boldsymbol{C}, \boldsymbol{F})$ animal but not of the wild type $(\boldsymbol{A}, \boldsymbol{C})$. FP, Floor plate; MN, motor neurons. Scale bar, $50 \mu \mathrm{m}$.
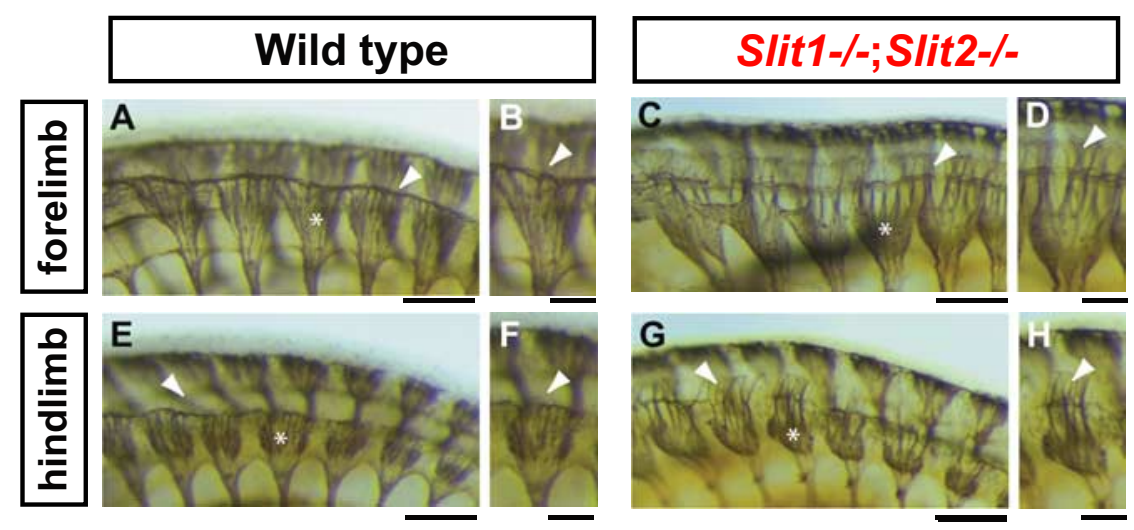

\section{Robo1+/-;Robo2+/-}
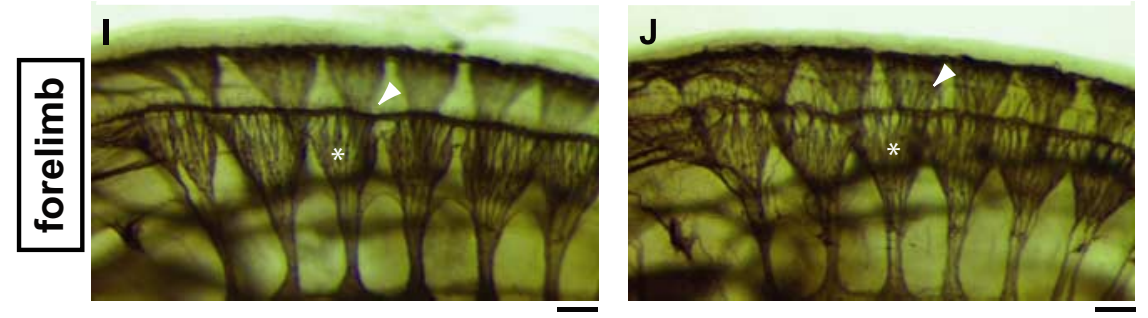

Figure 4. Whole-mount neurofilament staining in E10.5 mouse mutants. Sensory axonal projections are visualized by neurofilament immunostaining in the whole-mount preparation, which reveals all of the peripheral nerves in E10.5 embryos. Embryos were viewed from the dorsolateral side of the body, where individual DRGs as well as the bifurcated branches that form the longitudinal tracts at the DREZ (arrows) can be seen easily. In the wild-type $(\boldsymbol{A})$ or the $R o b 01^{+/-} ; R_{0 b 02}{ }^{+/-}(\boldsymbol{I})$ embryo, the space (arrowheads) between the left and right dorsal funiculi is devoid of any labeled neurites. However, in either the Slit ${ }^{-/-} ; S$ Slit $2^{-/}$

$(\boldsymbol{C})$ or the $R o b 01^{-/-} ; R o b 02^{-/-}(\boldsymbol{J})$ animal, this space is populated by overshooting fibers that grow out from the DRG $\left(^{*}\right)$. In addition, the overshooting fibers can be seen in the hindlimb region ( $G$ vs $\boldsymbol{E}$ ), where sensory afferents just reach the DRZ and start to bifurcate. Higher magnification views of sensory afferents at the DREZ are shown in $\boldsymbol{B}, \boldsymbol{D}, \boldsymbol{F}$, and $\boldsymbol{H}$. Scale bars: $\boldsymbol{A}, \boldsymbol{C}, \boldsymbol{E}, \boldsymbol{G}, \boldsymbol{I}, \boldsymbol{J}, 100$ $\mu \mathrm{m} ; \boldsymbol{B}, \boldsymbol{D}, \boldsymbol{F}, \boldsymbol{H}, 50 \mu \mathrm{m}$.

be best visualized by neurofilament immunostaining in wholemount embryos (Fig. 4). Although the bundled axonal tracts at the DREZ still form in the Slit $1^{-1-} ;$ Slit $^{-1-}$ or Robo1 $1^{-1-}$; Robo2 $2^{-1-}$ embryos $(n>3)$, many fibers leave the longitudinal

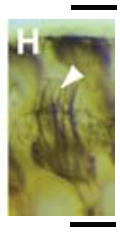

tract and fill in the normally clear space between the left and right DREZs (Fig. $4 A, C, E, G, I, J$, arrowheads). Consistent with what is seen from the DiI labeling in E13.5 embryos (Fig. 2) and immunostaining in cross-sections (Fig. 3), overshooting fibers project toward the midline, and some of them appear to bifurcate and form a secondary tract near the ventricular zone, leaving a clear but narrow space between the two normal dorsal funiculi (Fig. $4 C, G, J)$. In addition, the dorsal funiculus becomes defasciculated, and individual fibers could be seen in the longitudinal tract. Because of the developmental delay along the rostrocaudal axis, we could also easily find transition regions near the hindlimb where the sensory axons had just reached the spinal cord (Fig. $4 B, D, F, H$, arrows). In these regions, we found that the overshooting fibers were always associated with the appearance of the dorsal funiculus, suggesting that the defects occur at the time of bifurcation.

The gene dosage dependence of this phenotype is obvious in the whole-mount analysis. When three of the four alleles of Slit 1 and Slit 2 were removed, a small number of misprojections were observed, although the extent of the defect was at least fourfold less that in the double mutants (data not shown). This correlation was consistent with DiI labeling, which revealed a profound defect in the Slit ${ }^{-/-}$; Slit $2^{-/-}$double mutant but a smaller but still clear defect in Slit ${ }^{+/-} ;$Slit $2^{-/-}$embryos (Fig. 2G). Therefore, Slit1 and Slit2 are functionally redundant in controlling these sensory axon projections, and a single copy of either gene is mostly but not entirely sufficient to prevent misprojections.

Together, these results suggest that the misprojection does not result from premature formation of collaterals, and instead, Slit/Robo signaling plays a significant role in controlling some aspects of sensory axon growth or guidance during bifurcation.

\section{Single-cell analysis of bifurcation}

To understand how bifurcation is affected by loss of Slit/Robo signaling, we directly visualized the projection pattern of aberrant axons with single-cell resolution by iontophoresis of small amounts of DiI into the DRG in an open book preparation. The primary central axons in wildtype E13.5 embryos usually bifurcate to form T- or Y-shaped forks at the DREZ, which can be easily visualized from the lateral side of the spinal cord (Fig. 5A-C). In Slit $1^{-/-} ;$Slit $2^{-/-}$mutants, all of the axons still bifurcated. Approximately half of them formed normal bifurcation forks (Fig. 
$5 A, B)$, but in the other half, one of the daughter branches, either rostral or caudal, failed to make a right angle turn to extend longitudinally (Fig. 5D-G, arrows, $H)$. Instead, this branch aberrantly continued extending in the same direction as the primary axon and grew straight into the spinal cord and toward the midline. These aberrant paths can be well visualized using stereo images reconstructed from confocal sections (supplemental Fig. 7, available at www.jneurosci.org as supplemental material). In the double mutants, some of the axons were also kinked (Fig. 5D-F, arrowheads). Finally, the same defect was also seen in the Robo1;Robo2 double mutant (data not shown).

This behavior of axons at the single-cell level is consistent with the population behavior seen in sections (Figs. 2, 3) and whole-mount preparations of the spinal cord (Fig. 4) and suggests that the normal role of Slit proteins is to guide the branches during bifurcation, rather than to regulate bifurcation per se. A simple model would be that, at these early stages, Slit proteins negatively regulate the entry of one of the branches into the spinal cord, confining it to the dorsal funiculus and ensuring that it courses rostrocaudally.

\section{Slit2N negatively regulates sensory axon growth in vitro}

Based on these results, we therefore examined whether Slit proteins can negatively regulate sensory axon growth or guidance in vitro. Our previous studies demonstrating that the positive branching effect of Slit2N was performed with E14 rat sensory neurons (developmentally equivalent to E12.5 in the mouse), but the guidance of central branches at the DREZ occurs $\sim 2 \mathrm{~d}$ earlier. We therefore examined the activity of Slit2N on E12 rat sensory neurons (developmentally equivalent to E10.5 mouse cells). We initially focused on an in vitro repulsion assay in which DRG explants grown with either NGF or NT3 (to elicit growth of different afferent populations) are cocultured for $40 \mathrm{~h}$ with COS cell aggregates secreting Slit2N in a collagen gel matrix (Messersmith et al., 1995); however, no effect of Slit $2 \mathrm{~N}$ was seen in this assay, and the sensory axons grew out radially in a halo (data not shown).

Because we did not see any activity in this chronic coculture assay, we next turned to a more transient growth cone collapse assay (Luo et al., 1993), in which E12 rat sensory neurons were cultured with either NGF or NT-3 and exposed acutely to Slit2N proteins. We initially did not find any activity either, when we followed traditional protocols to examine the growth cone morphology after 45-60 min of treatment of Slit2N. However, after we followed the time course carefully, we did find that Slit $2 \mathrm{~N}$ caused growth cones of both neuron types to collapse (Fig. $6 A, B)$. The collapse was, however, transient and relatively weak, at least when compared with that of the well known collapsing factor Sema3A. Approximately $60-70 \%$ of growth cones arrested growth $6-15$ min after the addition of S2N, but approximately half of them recovered 15 min later, whereas nearly all growth cones remained in the collapsed state when treated with Sema3A. This transient response can also be seen in time-lapse movies for both NGF $(n=4)$ and NT-3 $(n=5)$ cells. As shown in Figure 6C, we observed that the growth cones initially lost their fan shape and stalled but then regained their structure and motility. This result shows that Slits can have a negative effect, albeit transient, in regulating sensory axon growth at the time of axonal bifurcation at the DREZ.

\section{Discussion}

During the development of nerve connections, neurons not only regulate the growth and guidance of their axons and dendrites but also need to determine where and when to make branches. Sometimes they form only two branches, but other times they extend multiple branches from the same shaft or have exuberant branched arbors. Although it is clear that branching is regulated by both intrinsic programs as well as extrinsic factors, how di- 


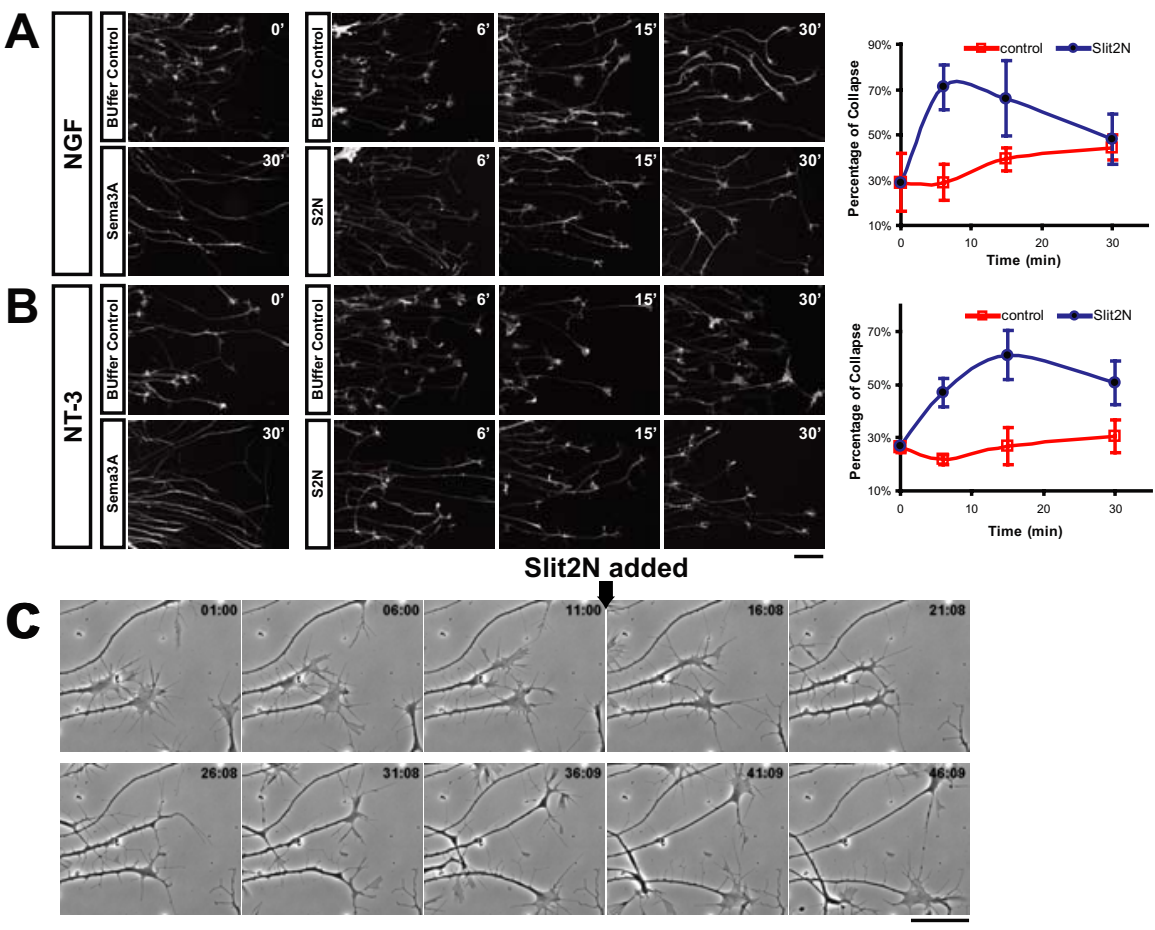

Figure 6. Slit2N induces transient collapse of young sensory axon growth cones in culture. $A, B, E 12$ rat DRG neurons cultured in the presence of NGF $(\boldsymbol{A})$ or NT-3 $(\boldsymbol{B})$ were stained with rhodamine-phalloidin to reveal the growth cones after the treatment of buffer, Slit2N, or Sema3A for different time duration (0,6, 15, and $30 \mathrm{~min})$. The percentage of growth cones that have lost their fan shape from cultures treated with buffer control and Slit2N is plotted for each time point. Scale bar, $100 \mu \mathrm{m}$. C, Sequential phase contrast images of E12 rat sensory growth cones in culture taken from a time lapse movie. The growth cones were followed for $\sim 11$ min before the addition of Slit2N to the medium (arrow). Note the reduction of growth cone size but no loss of filopodia between 21 and $31 \mathrm{~min}$ and the recovery of growth cones after $36 \mathrm{~min}$. Scale bar, $40 \mu \mathrm{m}$.

verse branched morphologies are generated is still poorly understood. Our results show that a single extrinsic factor can help sculpt different branched morphologies via distinct actions. This dual function helps understand how a limited set of factors are capable of generating scores of complex branching patterns in the developing brain.

\section{Slit/Robo signaling regulates terminal arborization of peripheral sensory projections}

Slits were originally identified based on both their repulsive effects on axons (Brose et al., 1999; Kidd et al., 1999; Li et al., 1999) and their ability to promote sensory axon elongation and branch formation (Wang et al., 1999). Here, we provide genetic evidence to show that the positive activity of Slits is used to control some peripheral arbors of sensory neurons in vivo. In mice lacking functional Slit2 and Slit3, we found a striking reduction in one of the ophthalmic branches during trigeminal development. This is consistent with a gain-of-function study in which overexpression of Slit in zebrafish somites induced branching of peripheral nerves (Yeo et al., 2004). Interestingly, in younger embryos, the branches are completely absent from the mutant compared with the control (supplemental Fig. 3B, available at www.jneurosci.org as supplemental material), whereas in embryos that are a few hours older, the branches emerge but with much smaller and delayed pattern (Fig. $1 F$ ). This result suggests that the Slit signal is used to promote initial branch formation when peripheral projections just reach the skin. It also suggests that additional factors, presumably including neurotrophins, also help them assume their mature patterns. In addition, whereas the Slits are limiting for the development of the ophthalmic branch of the trigeminal projections, they do not appear to be limiting for the other trigeminal branches or for spinal sensory projections, which are primarily normal in these embryos. The other factors that contribute to the branching of those peripheral projections remain to be determined.

Our results also provide in vivo evidence that Robo receptors mediate the positive branching activity of Slits, because the Robol;Robo2 double mutant mice have the same peripheral branching phenotype as Slit triple mutants in the trigeminal system. Again, this is the first genetic evidence to show that Robos are involved in the positive branching activity of Slits. However, this finding raises the question of how the same receptor can participate in both repulsion and branching in vivo. Additional studies of Robo signaling will help address this question.

\section{Slit/Robo signaling is also required for} proper bifurcation of central afferents We also studied the central afferent branching of sensory neurons, because Slits have been proposed to control central collateral formation (Wang et al., 1999) and have been shown to stimulate trigeminal ganglia to sprout collaterals into the brainstem in an organotypic culture (Ozdinler and Erzurumlu, 2002). However, we did not observe any clear defects in the formation of central collateral branches inside the spinal cord. Both the cutaneous and the muscle afferents are formed and enter the spinal cord, projecting to appropriate laminas (Fig. 2) (supplemental Fig. 4, available at www.jneurosci.org as supplemental material). However, because our DiI analysis only reveals the bulk behavior of the afferents, we cannot exclude the possibility that there are minor errors, or that some specific subtypes of collaterals might be regulated by Slits.

An unexpected finding from our analysis of central afferents is that their initial bifurcation outside the spinal cord gray matter at earlier stages is regulated by Slit/Robo signaling. In Robo1;Robo2 or Slit1;Slit2 double mutants, the central afferents of DRG cells no longer form the normal T-shape bifurcation fork (Fig. 5). Instead, one of the branches is misguided and enters the spinal cord. Together with the observation that both Slit1 and Slit2 (but not Slit3) are expressed in the superficial layer of the lateral spinal cord at the time of bifurcation, this phenotype suggests that Slit/ Robo signaling is normally used to keep the sensory afferents outside the spinal cord at that stage, presumably through repulsion. A function in repulsion is further supported by the growth cone collapsing activity we observed for Slit $2 \mathrm{~N}$ on young sensory axons in vitro. Interestingly, this collapsing activity is transient. Our in vivo and in vitro results together support a model in which Slit/Robo signaling negatively regulates the growth direction of one daughter branch during bifurcation in vivo; the fact that the collapsing activity of Slits on these axons is transient seems appropriate given the need to ensure the growth cone reorients growth and grows alongside the source of Slit after being de- 
flected away from the dorsal spinal cord. Importantly, the axons always form two branches, although one is misguided, suggesting that cues other than the Slits are responsible for instructing the axon to bifurcate, with Slit/Robo signaling only affecting the direction of guidance of one branch. Why a single branch is affected remains to be determined, but this result suggests that the two branches that result from the bifurcation are not equivalent in their complement of axon guidance signaling pathways. Additional studies will be required to identify the factors that collaborate with Slits to regulate bifurcation and branch guidance in the DREZ region.

\section{Bifunctional roles of Slits in controlling sensory axon branching}

How Slits, acting via Robos, elicit these distinct responses in sensory axons remains to be determined. Secreted axon guidance factors can both positively and negatively regulate nerve growth and guidance, depending on the receptors that are expressed or the signaling mechanisms active in the neurons, including the level of cyclic nucleotides (Tessier-Lavigne and Goodman, 1996; Dickson, 2002). The distinct roles of Slits on the peripheral and central branches of sensory axons are reminiscent, at least at a gross level, of the distinct activities of the Semaphorin (Sema3A) on the dendrites and axons of cortical neurons: Sema3A repels the axon but stimulates branching of the dendrites, a parallel that is all the more intriguing, because the peripheral branch of sensory neurons may, at some level, be compared with a dendrite. In the case of cortical neurons, the difference in responses has been attributed to, at least in part, a higher level of cGMP in dendrites compared with the axons that is postulated to result from the enrichment in dendrites of guanylate cyclase (Polleux et al., 2000). In fact, cGMP has been shown to be able to modulate the response of growth cones to Slits (Nguyen-Ba-Charvet et al., 2001). Future studies will help determine whether this mechanism indeed contributes to the difference between the responses of central and peripheral branches. Regardless of mechanism, however, our studies emphasize the versatility of the Slit/Robo signaling system in vivo, which appears to be deployed in multiple ways to control distinct aspects of axon guidance and branching.

\section{References}

Bagri A, Cheng HJ, Yaron A, Pleasure SJ, Tessier-Lavigne M (2003) Stereotyped pruning of long hippocampal axon branches triggered by retraction inducers of the semaphorin family. Cell 113:285-299.

Brose K, Bland KS, Wang KH, Arnott D, Henzel W, Goodman CS, TessierLavigne M, Kidd T (1999) Slit proteins bind Robo receptors and have an evolutionarily conserved role in repulsive axon guidance. Cell 96:795-806.

Cheng HJ, Bagri A, Yaron A, Stein E, Pleasure SJ, Tessier-Lavigne M (2001) Plexin-a3 mediates semaphorin signaling and regulates the development of hippocampal axonal projections. Neuron 32:249-263.

Cohen-Cory S, Fraser SE (1995) Effects of brain-derived neurotrophic factor on optic axon branching and remodelling in vivo. Nature 378:192-196.

Dickson BJ (2002) Molecular mechanisms of axon guidance. Science 298:1959-1964

Dodd J, Morton SB, Karagogeos D, Yamamoto M, Jessell TM (1988) Spatial regulation of axonal glycoprotein expression on subsets of embryonic spinal neurons. Neuron 1:105-116.

Fricke C, Lee JS, Geiger-Rudolph S, Bonhoeffer F, Chien CB (2001) astray, a zebrafish roundabout homolog required for retinal axon guidance. Science 292:507-510.

Furley AJ, Morton SB, Manalo D, Karagogeos D, Dodd J, Jessell TM (1990) The axonal glycoprotein TAG-1 is an immunoglobulin superfamily member with neurite outgrowth-promoting activity. Cell 61:157-170.
Grieshammer U, Le M, Plump AS, Wang F, Tessier-Lavigne M, Martin GR (2004) SLIT2-mediated ROBO2 signaling restricts kidney induction to a single site. Dev Cell 6:709-717.

Hao JC, Yu TW, Fujisawa K, Culotti JG, Gengyo-Ando K, Mitani S, Moulder G, Barstead R, Tessier-Lavigne M, Bargmann CI (2001) C. elegans slit acts in midline, dorsal-ventral, and anterior-posterior guidance via the SAX-3/Robo receptor. Neuron 32:25-38.

Hoyle GW, Mercer EH, Palmiter RD, Brinster RL (1993) Expression of NGF in sympathetic neurons leads to excessive axon outgrowth from ganglia but decreased terminal innervation within tissues. Neuron 10:1019-1034

Kennedy TE, Serafini T, de la Torre JR, Tessier-Lavigne M (1994) Netrins are diffusible chemotropic factors for commissural axons in the embryonic spinal cord. Cell 78:425-435.

Kidd T, Brose K, Mitchell KJ, Fetter RD, Tessier-Lavigne M, Goodman CS, Tear G (1998) Roundabout controls axon crossing of the CNS midline and defines a novel subfamily of evolutionarily conserved guidance receptors. Cell 92:205-215.

Kidd T, Bland KS, Goodman CS (1999) Slit is the midline repellent for the robo receptor in Drosophila. Cell 96:785-794.

Krylova O, Herreros J, Cleverley KE, Ehler E, Henriquez JP, Hughes SM, Salinas PC (2002) WNT-3, expressed by motoneurons, regulates terminal arborization of neurotrophin-3-responsive spinal sensory neurons. Neuron 35:1043-1056.

Lentz SI, Knudson CM, Korsmeyer SJ, Snider WD (1999) Neurotrophins support the development of diverse sensory axon morphologies. J Neurosci 19:1038-1048.

Li HS, Chen JH, Wu W, Fagaly T, Zhou L, Yuan W, Dupuis S, Jiang ZH, Nash W, Gick C, Ornitz DM, Wu JY, Rao Y (1999) Vertebrate slit, a secreted ligand for the transmembrane protein roundabout, is a repellent for olfactory bulb axons. Cell 96:807-818.

Livet J, Sigrist M, Stroebel S, De Paola V, Price SR, Henderson CE, Jessell TM, Arber S (2002) ETS gene Pea3 controls the central position and terminal arborization of specific motor neuron pools. Neuron 35:877-892.

Long H, Sabatier C, Ma L, Plump A, Yuan W, Ornitz DM, Tamada A, Murakami F, Goodman CS, Tessier-Lavigne M (2004) Conserved roles for Slit and Robo proteins in midline commissural axon guidance. Neuron 42:213-223.

Luo Y, Raible D, Raper JA (1993) Collapsin: a protein in brain that induces the collapse and paralysis of neuronal growth cones. Cell 75:217-227.

Messersmith EK, Leonardo ED, Shatz CJ, Tessier-Lavigne M, Goodman CS, Kolodkin AL (1995) Semaphorin III can function as a selective chemorepellent to pattern sensory projections in the spinal cord. Neuron 14:949-959.

Nguyen-Ba-Charvet KT, Brose K, Marillat V, Sotelo C, Tessier-Lavigne M, Chedotal A (2001) Sensory axon response to substrate-bound Slit2 is modulated by laminin and cyclic GMP. Mol Cell Neurosci 17:1048-1058.

Ozaki S, Snider WD (1997) Initial trajectories of sensory axons toward laminar targets in the developing mouse spinal cord. J Comp Neurol 380:215-229.

Ozdinler PH, Erzurumlu RS (2002) Slit2, a branching-arborization factor for sensory axons in the mammalian CNS. J Neurosci 22:4540-4549.

Parrish JZ, Kim MD, Jan LY, Jan YN (2006) Genome-wide analyses identify transcription factors required for proper morphogenesis of Drosophila sensory neuron dendrites. Genes Dev 20:820-835.

Plump AS, Erskine L, Sabatier C, Brose K, Epstein CJ, Goodman CS, Mason CA, Tessier-Lavigne M (2002) Slit1 and Slit2 cooperate to prevent premature midline crossing of retinal axons in the mouse visual system. Neuron 33:219-232.

Polleux F, Morrow T, Ghosh A (2000) Semaphorin 3A is a chemoattractant for cortical apical dendrites. Nature 404:567-573.

Sabatier C, Plump AS, Ma L, Brose K, Tamada A, Murakami F, Lee EY, Tessier-Lavigne M (2004) The divergent Robo family protein rig-1/ Robo3 is a negative regulator of slit responsiveness required for midline crossing by commissural axons. Cell 117:157-169.

Serafini T, Colamarino SA, Leonardo ED, Wang H, Beddington R, Skarnes WC, Tessier-Lavigne M (1996) Netrin-1 is required for commissural axon guidance in the developing vertebrate nervous system. Cell 87:1001-1014.

Soussi-Yanicostas N, de Castro F, Julliard AK, Perfettini I, Chedotal A, Petit C (2002) Anosmin-1, defective in the X-linked form of Kallmann syn- 
drome, promotes axonal branch formation from olfactory bulb output neurons. Cell 109:217-228.

Tessier-Lavigne M, Goodman CS (1996) The molecular biology of axon guidance. Science 274:1123-1133.

Wang KH, Brose K, Arnott D, Kidd T, Goodman CS, Henzel W, TessierLavigne M (1999) Biochemical purification of a mammalian slit protein as a positive regulator of sensory axon elongation and branching. Cell 96:771-784

Whitford KL, Marillat V, Stein E, Goodman CS, Tessier-Lavigne M, Chedotal A, Ghosh A (2002) Regulation of cortical dendrite development by SlitRobo interactions. Neuron 33:47-61.

Yates PA, Roskies AL, McLaughlin T, O’Leary DD (2001) Topographic- specific axon branching controlled by ephrin-As is the critical event in retinotectal map development. J Neurosci 21:8548-8563.

Yeo SY, Miyashita T, Fricke C, Little MH, Yamada T, Kuwada JY, Huh TL, Chien CB, Okamoto H (2004) Involvement of Islet-2 in the Slit signaling for axonal branching and defasciculation of the sensory neurons in embryonic zebrafish. Mech Dev 121:315-324.

Yuan W, Rao Y, Babiuk RP, Greer JJ, Wu JY, Ornitz DM (2003) A genetic model for a central (septum transversum) congenital diaphragmatic hernia in mice lacking Slit3. Proc Natl Acad Sci USA 100:5217-5222.

Zallen JA, Yi BA, Bargmann CI (1998) The conserved immunoglobulin superfamily member SAX-3/Robo directs multiple aspects of axon guidance in C. elegans. Cell 92:217-227. 